\section{NEW APP SHOWS AMALGAM/COMPOSITE CAVITY DESIGNS}

A groundbreaking new dental app has been released by DentalAppCo in conjunction with academics from the University of Manchester and the University of Sheffield. The dental app uses modified high resolution micro-CT scan of real teeth to show various cavity designs for amalgam and composite filling materials.

The app was designed to be used within a teaching environment to enable students to study and visualise cavity preparation. The teeth can be rotated, zoomed and moved around so that the cavity designs can be examined closely and teaching staff can point out various features. Students can gain a solid 3D image of what is required, and this will help them to understand the principles of tooth preparation.

For more

information see www. dentalappco.com/ cavsim.

iPhone/iPad link: itunes.apple. com/app/id934845652

Android link: play.google. com/store/apps/details?id=com. fungameco.filling

\title{
HELPING INDIVIDUALS RETAIN THEIR ENAMEL
}

The consumption of soft drinks, fruit juices and sports drinks continue to rise. The erosive nature of these drinks is well documented, which begs the question of what can be done to provide protection against their damaging effect. One study found that toothpaste containing stabilised stannous fluoride provided significant acid protection over that provided by conventional fluoride products. This is strongly attributable to the high bioavailability of stannous fluoride in the formula. It is believed that toothpastes containing this ingredient produce a protective barrier layer that remains on the tooth for hours after the product's use. It is this, the authors conclude, which helps protect enamel against the initiation and progression of dietary acid attack.
Oral-B Pro-Expert toothpaste contains a combination of stabilised stannous fluoride and sodium hexametaphosphate. Preventing enamel loss is just one benefit of Pro-Expert. It also helps protect against plaque, gum problems, caries, calculus formation, dental hypersensitivity, staining and oral malodour.

Whilst it is hoped that education on the damaging effects of acidic drinks will go some way in controlling the problem of erosion, providing protection against the effect of such drinks will help individuals retain their enamel. Unlike the supply of these damaging beverages, once enamel's gone, it's gone!

1. Faller R V, Eversole S L, Tzeghai G E. Enamel protection: a comparison of marketed dentifrice performance against dental erosion. Am J Dent 2011; 24: 205-210.

\section{INVISIBLE PROTECTION}

\section{Clinpro Fissure Sealant from}

3M ESPE is proven to provide longlasting protection against caries.

This is the first fissure sealant with colour-change technology. Accurate and even application is easy thanks to its pink hue, which transforms into an opaque white after curing. It also flows easily into pits and fissures thanks to its ultrafine syringe tip and low viscosity.

Contact 3M ESPE to find out how Clinpro Fissure Sealant could benefit your patients on 08456025094 or visit www.3Mespe.co.uk.

3M, ESPE and Clinpro are trademarks of the 3M Company.

\section{PART OF AN EFFECTIVE PERIODONTAL TREATMENT PROGRAMME}

Periodontitis can destroy teeth and damage gingival tissue, but it has also been associated with other serious health issues such as respiratory diseases.

The treatment of periodontal disease often begins with root planing and scaling, but in certain cases, adjunctive treatment is required. For patients with pocketing of $5 \mathrm{~mm}$ or more, PerioChip is a clinically effective option and achieves better results when combined with your RSD.

The wafer thin, biodegradable insert contains $36 \%$ chlorhexidine digluconate and takes just 30 seconds to place in the periodontal pocket; once placed it kills 99\% of bacteria for up to ten days. The antimicrobial effects from placing a PerioChip continue to inhibit bacterial flora for up to 11 weeks thereafter for long lasting results.

To help your patients in the battle against periodontal disease and the potential myriad other associated health problems, provide PerioChip as part of an effective periodontal treatment programme.

To order PerioChip or for more information, email team@periochip.co.uk or freephone 0800013233.

\section{LOOK, NO STAINS}

When gingivitis or periodontal disease strike, Curasept Anti-Discolouration System (ADS) mouthwash provides an effective solution for all your patients.

From Curaprox, the long-lasting mouthwash contains the active ingredient chlorhexidine (CHX), killing bacteria fast, while avoiding all of the traditional side effects.

As a result, the mouthwash prevents red and swollen gums and reduces bleeding, bad breath and tooth decay, without causing any staining to the teeth or tongue, mouth irritation or changes in taste sensation.

Being alcohol-free, patients can also use Curasept ADS without concern for any possible links between alcohol in mouthwashes and oral cancer.

Cost effective and available in three different strengths $(0.2 \%$, 0.12\% and 0.05\%), Curasept ADS can be used with a complementary gel and SLS-free toothpaste.

Recommend Curasept ADS

to your patients today and help them achieve long-lasting oral health.

For more information call 01480 862084 or visit www.curaprox.co.uk. 